\title{
RANDOM FIXED POINT THEOREMS ON PRODUCT SPACES ${ }^{1}$
}

\author{
ISMAT BEG ${ }^{2}$ and NASEER SHAHZAD \\ Quaid-i-Azam University \\ Department of Mathematics \\ Islamabad 45320, PAKISTAN
}

\begin{abstract}
The existence of random fixed point of a locally contractive random operator in first variable on product spaces is proved. The concept "continuous random height-selection" is discussed. Some random fixed point theorems for nonexpansive self and nonself maps are also obtained in product spaces.
\end{abstract}

Key words: Product space, random operator, random fixed point, weakly inward operator.

AMS (MOS) subject classifications: $47 \mathrm{H} 10,60 \mathrm{H} 25,54 \mathrm{H} 25$, $47 \mathrm{H} 40,47 \mathrm{H} 09$.

\section{INTRODUCTION}

The study of the fixed points of random operators of various types is a lively and fascinating field of research lying at the intersection of nonlinear analysis and probability theory. A wide spread interest in the domain and a vast amount of mathematical activity have led to many remarkable new results and viewpoints yielding insight even into traditional question.

Random fixed point theorems are stochastic generalizations of classical fixed point theorems. In Polish spaces, random fixed point theorems for contraction mappings were proved by Spacek [24] and Hans [7, 8]. For a complete survey, we refer to Bharucha-Reid [2, 3]. Itoh [10, 11, 12] gave several random fixed point theorems for various single and multivalued random operators, that is, $\alpha$-condensing or nonexpansive random operators. He also gave several common random fixed point theorems for commuting random operators. Afterwards, Sehgal and Singh [23], Papageorgiou [22] and Lin [20] gave different stochastic versions of a very interesting approximation theorem of Fan [5]. Recently, Beg and Shahzad

\footnotetext{
${ }^{1}$ Received: August, 1992. Revised: February, 1993.

${ }^{2}$ Research supported by National Scientific Research and Development Board grant No.
} M.Sc.Sc. (5)/QAU/90. 
[1] studied the structure of common random fixed points and random coincidence points of a pair of compatible random multivalued operators in Polish spaces. They also proved a random fixed point theorem for contractive random operators in 6 -chainable Polish spaces. An interesting application to random approximation is also given. The aim of this paper is to prove several random fixed point theorems for various self and nonself random operators, that is, contractive and nonexpansive random operators in product spaces. The concept "continuous random height-selection" is discussed and its relation to the existence of random fixed points for a function is shown. Section 2 contains definitions and preliminary material. In section 3 , the existence of random fixed point of a contractive random operator in first variable on product spaces is proved. Other results are proved concerning the random fixed point theorem for product spaces. Section 4 contains some random fixed point theorems for nonexpansive self or nonself random operators.

\section{PRELIMINARIES}

Throughout this paper, let $(X, d)$ be a Polish space, that is, a separable complete metric space, and $(\Omega, \mathcal{A})$ be a measurable space. Let $2^{X}$ be the family of all subsets of $X$ and $W K(X)$ the family of all weakly compact subsets of $X$. A mapping $T: \Omega \rightarrow 2^{X}$ is called measurable if for any open subset $C$ of $X, T^{-1}(C)=\{\omega \in \Omega: T(\omega) \cap C \neq \phi\} \in \mathcal{A}$. This type of measurability is usually called weak measurability (cf. Himmelberg [9]), but in this paper since we only use this type of measurability, thus we omit the term "weak" for simplicity. A mapping $\zeta: \Omega \rightarrow X$ is said to be the measurable selector of a measurable mapping $T: \Omega \rightarrow 2^{X}$ if $\zeta$ is measurable and for any $\omega \in \Omega, \zeta(\omega) \in T(\omega)$. A mapping $f: \Omega \times X \rightarrow X$ is called a random operator if for any $x \in X, f(\cdot, x)$ is measurable. A measurable mapping $\zeta: \Omega \rightarrow X$ is called random fixed point of a random operator $f: \Omega \times X \rightarrow X$ if for every $\omega \in \Omega, f(\omega, \zeta(\omega))=\zeta(\omega)$. A random operator $f: \Omega \times X \rightarrow X$ is called continuous if for each $\omega \in \Omega, f(\omega, \cdot)$ is continuous. A random operator $T: \Omega \times X \rightarrow X$ is said to be nonexpansive if for any $\omega \in \Omega$, $\|T(\omega, u)-T(\omega, v)\| \leq\|u-v\|$ for all $u, v \in X$.

A separable metric space $Y$ has a random fixed point property for nonexpansive (continuous) random operators if every nonexpansive (continuous) random operator $T: \Omega \times Y \rightarrow Y$ must have a random fixed point. In what follows, $P_{1}: X \times Y \rightarrow X$ will denote the first projection mapping defined by $P_{1}(x, y)=x$, while $P_{2}: X \times Y \rightarrow Y$ will denote the second projection mapping defined by $P_{2}(x, y)=y$. 


\section{RANDOM FIXED POINT THEOREMS AND CONTINUOUS RANDOM HEIGHT-SELECTION}

In 1968, Nadler [21] established some fixed point theorems for uniformly continuous functions on product metric spaces. Subsequently, Fora [6] further generalized these results. Recently, Kuczumow [18] proved a fixed point theorem in product spaces using the generic fixed point property for nonexpansive mapping. The aim of this section is to prove some random fixed point theorems using a recent result of Beg and Shahzad [1].

Let $X$ be a Polish space and $Y$ be any space. A random operator $f: \Omega \times(X \times Y) \rightarrow(X \times Y)$ is said to be a locally contractive random operator in the first variable if and only if for any $x_{*} \in X$ there exists $\epsilon>0$ and a measurable map $\lambda: \Omega \rightarrow(0,1)$ such that $p, q \in S\left(x_{*}, \epsilon\right)=\left\{z \in X: d\left(x_{*}, z\right)<\epsilon\right\}$ and for any $\omega \in \Omega$

$$
d\left(P_{1} \circ f(\omega, p, y), P_{1} \circ f(\omega, q, y)\right) \leq \lambda(\omega) d(p, q)
$$

for any $y \in Y$.

A random operator $f: \Omega \times(X \times Y) \rightarrow(X \times Y)$ is called a contraction mapping in the first variable if and only if there exists a measurable map $\lambda: \Omega \rightarrow(0,1)$ such that for any $y \in Y$, we have for any $\omega \in \Omega$

$$
d\left(P_{1} \circ f(\omega, x, y), P_{1} \circ f\left(\omega, x_{*}, y\right)\right) \leq \lambda(\omega) d\left(x, x_{*}\right)
$$

for all $x, x_{*} \in X$.

A metric space $(X, d)$ is said to be $\epsilon$-chainable if and only if given $x, y$ in $X$, there is an $\epsilon$-chain from $x$ to $y$ (i.e., a finite set of points $x=z_{0}, z_{1}, \ldots, z_{n}=y$ such that $d\left(z_{j-1}, z_{j}\right)<\epsilon$ for $j=1,2, \ldots, n)$.

Theorem 3.1: $\quad$ Let $X$ be an $\epsilon$-chainable Polish space, let $Y$ be a separable metric space with the random fixed point property and let $f: \Omega \times(X \times Y) \rightarrow X \times Y$ be a continuous random operator. If $f$ is a locally contractive operator in the first variable, then $f$ has a random fixed point.

Proof: Let $\xi_{0}: \Omega \rightarrow X$ be a fixed measurable mapping. Define the random operator $g: \Omega \times Y \rightarrow Y$ as follows. Let $y \in Y, \omega \in \Omega$. In order to define $g(\omega, y)$, we first define a sequence of measurable mappings $\left\{\eta_{n}(\omega, y)\right\}$ as follows

$$
\eta_{0}(\omega, y)=\xi_{0}(\omega), \eta_{n}(\omega, y)=P_{1} \circ f\left(\omega, \eta_{n-1}(\omega, y), y\right)
$$


$n=1,2, \ldots$ For simplicity, we shall write $\eta_{n}(\omega)$ to stand for $\eta_{n}(\omega, y)$. Since $f$ is a locally contractive random operator in the first variable, there exists a measurable map $\lambda: \Omega \rightarrow(0,1)$ such that

$$
d\left(P_{1} \circ f\left(\omega, \eta_{n-1}(\omega) y\right), P_{1} \circ f\left(\omega, \eta_{n}(\omega), y\right)\right) \leq \lambda(\omega) d\left(\eta_{n-1}(\omega), \eta_{n}(\omega)\right), n \geq 1 .
$$

By induction we find that

$$
d\left(\eta_{n}(\omega), \eta_{n+1}(\omega)\right) \leq \lambda^{n}(\omega) d\left(\eta_{0}(\omega), \eta_{1}(\omega)\right)
$$

It further implies that $\left\{\eta_{n}(\omega)\right\}$ is a Cauchy sequence. Since $X$ is complete, there exists $\eta_{y}(\omega) \in X$ such that $\eta_{n}(\omega) \rightarrow \eta_{y}(\omega)$ for each $\omega \in \Omega$. [The mapping $\eta_{y}: \Omega \rightarrow X$ is a point-wise limit of measurable mappings $\left\{\eta_{n}\right\}$, therefore measurable]. Thus we obtain $P_{1} \circ f\left(\omega, \eta_{y}(\omega), y\right)=\eta_{y}(\omega)$ for each $\omega \in \Omega$ (see Beg and Shahzad [1]). Now define for any $\omega \in \Omega$, $g(\omega, y)=P_{2} \circ f\left(\omega, \eta_{y}(\omega), y\right)$. Then $g$ is continuous [6, Lemma 3]. Since $Y$ has the random fixed point property, there is a measurable map $\xi: \Omega \rightarrow Y$ such that $g(\omega, \xi(\omega))=\xi(\omega)$ for each $\omega \in \Omega$. But $\xi(\omega)=g(\omega, \xi(\omega))=P_{2} o f\left(\omega, \eta_{\xi(\omega)}(\omega), \xi(\omega)\right) \quad$ for every $\omega \in \Omega$. We also have $P_{1} \circ f\left(\omega, \eta_{\xi(\omega)}(\omega), \xi(\omega)\right)=\eta_{\xi(\omega)}(\omega)$. Hence for $\omega \in \Omega, f\left(\omega, \eta_{\xi(\omega)}(\omega), \xi(\omega)\right)=\left(\eta_{\xi(\omega)}(\omega), \xi(\omega)\right)$.

Corollary 3.2: $\quad$ Let $X$ be a Polish space, let $Y$ be a separable metric space with random fixed point property and let $f: \Omega \times(X \times Y) \rightarrow(X \times Y)$ be a continuous random operator. If $f$ is a contraction random operator in the first variable, then $f$ has a random fixed point.

Let $f: \Omega \times(X \times Y) \rightarrow(X \times Y)$ be a continuous random operator. A measurable mapping $\eta_{x}: \Omega \rightarrow Y$ for which $P_{2} o f\left(\omega, x, \eta_{x}(\omega)\right)=\eta_{x}(\omega)$ for each $\omega \in \Omega$ is called a random fixed $f$-height of $x$. The set $\left\{\eta_{x}: \eta_{x}: \Omega \rightarrow Y\right.$ is a random $f$-height of $\left.x\right\}$ is called the random fixed $f$-height of $x$ and is denoted by $F(f, x)$. The set $U\{F(f, x): x \in X\}$ is called the random fixed $f$-height of $X$ and is denoted by $F(f, X)$. A continuous random height-selection of $f$ is a continuous random operator $g: \Omega \times X \rightarrow F(f, X)$ such that $g(\cdot, x): \Omega \rightarrow Y$ is a random $f$-height of $x$.

Theorem 3.3: $\quad$ Let $X$ and $Y$ be separable metric spaces with the random fixed point property, and let $f: \Omega \times(X \times Y) \rightarrow(X \times Y)$ be a continuous random operator. If $f$ has a continuous random height-selection, then $f$ has a random fixed point.

Proof: Let $x \in X$. Define a random operator $g^{\prime}: \Omega \times Y \rightarrow Y$ by the rule $g^{\prime}(\omega, y)=P_{2} \circ f(\omega, x, y)$. Then $g^{\prime}$ is continuous because $f$ is continuous. But $Y$ has the random fixed point property, therefore there exists a measurable mapping $\xi_{x}: \Omega \rightarrow Y$ such that $\xi_{x}(\omega)=g^{\prime}\left(\omega, \xi_{x}(\omega)\right)$ for each $\omega \in \Omega$. Thus $P_{2} \circ f\left(\omega, x, \xi_{x}(\omega)\right)=\xi_{x}(\omega)$, that is, $F(f, x) \neq \phi$. Let 
$g: \Omega \times X \rightarrow F(f, X)$ be a continuous random height selection of $f$. Define the continuous random operator $h: \Omega \times X \rightarrow X$ by the rule $h(\omega, x)=P_{1} o f(\omega, x, g(\omega, x))$ for each $\omega \in \Omega$ and $x \in X$. Let $\phi: \Omega \rightarrow X$ be a random fixed point of $h$. Then $h(\omega, \phi(\omega))=\phi(\omega)$ for $\omega \in \Omega$, that is, $P_{1} \circ f(\omega, \phi(\omega), g(\omega, \phi(\omega)))=\phi(\omega)$. Therefore $f(\omega, \phi(\omega), g(\omega, \phi(\omega)))=(\phi(\omega), v)$ for some $v \in Y$. But $g(\omega, \phi(\omega)) \in F(f, X)$ for each $\omega \in \Omega$. Therefore $P_{2} \circ f(\omega, \phi(\omega), g(\omega, \phi(\omega)))=g(\omega, \phi(\omega))$, that is, $f(\omega, \phi(\omega), g(\omega, \phi(\omega)))=(u, g(\omega, \phi(\omega)))$ for some $u \in X$. Hence for every $\omega \in \Omega, f(\omega, \phi(\omega)$, $g(\omega, \phi(\omega)))=(\phi(\omega), g(\omega, \phi(\omega)))$.

Using the proof of Theorem 3.1 and the technique used in the proof of Theorem 3.2, one can obtain the following theorem.

Theorem 3.4: Let $X$ be an $\epsilon$-chainable Polish space, let $Y$ be a separable metric space with the random fixed point property and let $f: \Omega \times(Y \times X) \rightarrow(Y \times X)$ be a continuous random operator. If $f$ is a locally contractive random operator in the second variable, then $f$ has a continuous random height-selection and hence $f$ has a random fixed point.

\section{RANDOM FIXED POINT THEOREMS OF NONEXPANSIVE RANDOM OPERATORS IN PRODUCT SPACES}

Let $E$ and $F$ be two Banach spaces with $X \subset E$ and $Y \subset F$. For $1 \leq P<\infty$ and $(x, y) \in E \oplus F$, set

$$
\|(x, y)\|_{p}=(\|x\| \underset{E}{p}+\|y\| \underset{F}{p})^{1 / p}
$$

and for $P=\infty,\|(x, y)\|_{\infty}=\max \left\{\|x\|_{E},\|y\|_{F}\right\}$.

It was shown in Kirk and Sternfeld [17] that if $E$ is uniformly convex (or reflexive with the B-G property), if $X$ is bounded closed convex, and if $Y$ is bounded closed and separable, then the assumption that $Y$ has the fixed point property for nonexpansive mappings assures the same is true of $(X \oplus Y)_{\infty}$. Subsequently, various results on fixed point theorems in product spaces were given by many authors (cf. Khamsi [13], Kirk [14, 15], Kirk and Yanez $[16]$ and their references).

Recently Tan and Xu [25] proved some fixed point theorems for nonexpansive self and nonself mappings in product spaces. They also generalized and improved some results of Kirk [15] and Kirk and Sternfeld [17]. In this section we give a stochastic version of the results of $[14,16,17,25]$. 
A subset $K$ of a Banach space $E$ has the Browder-Göhde (B-G) property [14] if for each nonexpansive mapping $T: K \rightarrow E$, the mapping (I-T) is demiclosed on $K$ (i.e., for each sequence $\left\{u_{j}\right\}$ in $K$, the condition $u_{j} \rightarrow u$ weakly and $u_{j}-T\left(u_{j}\right) \rightarrow p$ strongly implies $u \in K$ and $u-T(u)=p$ ). It is known (Browder [4]) that all closed convex subsets of uniformly convex Banach spaces have this property.

Theorem 4.1: $\quad$ Let $E$ and $F$ be two separable Banach spaces with $X \subset E$ and $Y \subset F$. Suppose that $X$ is weakly compact, convex, and has the B-G property. Suppose also that $Y$ has the random fixed point property for nonexpansive random operators. Then $(X \oplus Y)_{\infty}$ has the random fixed point property for nonexpansive random operators.

Proof: Let $P_{1}$ and $P_{2}$ be the natural projections of $(E \oplus F)$ onto $E$ and $F$, respectively. For each $y$ in $Y$, we define $T_{y}: \Omega \times X \rightarrow X$ by $T_{y}(\omega, x)=P_{1} o T(\omega, x, y), x \in X$. Then $T_{y}$ is a nonexpansive random operator. Let $S_{y}=\left(I+T_{y}\right) / 2(I$ denotes the identity operator on $E$ ). Let $\xi_{0}: \Omega \rightarrow X$ be a fixed measurable mapping. We have

$$
\lim _{n \rightarrow \infty}\left\|S_{y}\left(\omega, \xi_{y, n}(\omega)\right)-\xi_{y, n}(\omega)\right\|_{E}=0
$$

where $\xi_{y, n}=S_{y}^{n}\left(\omega, \xi_{0}(\omega)\right)=S_{y}\left(\omega, S_{y}^{n-1}\left(\omega, \xi_{0}(\omega)\right)\right.$, for each $\omega \in \Omega$. For each $n$, define $F_{y, n}: \Omega \rightarrow W K(X)$ by

$$
F_{y, n}(\omega)=w^{-c l}\left\{\xi_{y, i}(\omega): i \geq n\right\}
$$

where $w^{-c l}(C)$ is the weak closure of $C$.

Let $F_{y}: \Omega \rightarrow W K(X)$ be a mapping defined by $F_{y}(\omega)=\bigcap_{n=1}^{\infty} F_{y, n}(\omega)$. Then, since the weak-topology is a metric topology, $F_{y}$ is $w$-measurable by [9, Theorem 4.1]. Thus there is a $w$-measurable selector of $F_{y}[19]$. For any $x^{*} \in E^{*}$ (the dual space of $\left.E\right), x^{*}\left(\xi_{y}(\cdot)\right)$ is measurable as a numerically-valued function on $\Omega$. Since $E$ is separable, $\xi_{y}$ is measurable. Fix $\omega \in \Omega$ arbitrarily. Then some subsequences $\left\{\xi_{y, m}(\omega)\right\}$ of $\left\{\xi_{y, n}(\omega)\right\}$ converges weakly to $\xi_{y}(\omega)$. Then by the B-G property of $X$ and (1), it follows that $\xi_{y}(\omega)$ is a random fixed point of $S_{y}$ and hence of $T_{y}$, that is, $P_{1} \circ T\left(\omega, \xi_{y}(\omega), y\right)=\xi_{y}(\omega)$. Also,

$$
\left\|\xi_{u, n}(\omega)-\xi_{v, m}(\omega)\right\|_{E} \leq\|u-v\|_{F}, \quad n, m=1,2, \ldots
$$

for $u, v$ in $Y$ and $\omega \in \Omega$.

It further implies

$$
\left\|\xi_{u}(\omega)-\xi_{v}(\omega)\right\|_{E} \leq \lim _{n \rightarrow \infty} \sup \left[\lim _{m \rightarrow \infty} \sup \left\|\xi_{u, n}(\omega)-\xi_{v, m}(\omega)\right\|_{E}\right] \leq\|u-v\|_{F}
$$


Now let $f: \Omega \times Y \rightarrow Y$ defined by $f(\omega, y)=P_{2} o T\left(\omega, \xi_{y}(\omega), y\right), y \in Y$. Then for $u, v$ in $Y$, we have for any $\omega \in \Omega$

$$
\begin{gathered}
\|f(\omega, u)-f(\omega, v)\|_{F}=\left\|P_{2} o T\left(\omega, \xi_{u}(\omega), u\right)-P_{2} o T\left(\omega, \xi_{v}(\omega), v\right)\right\|_{F} \\
\leq\left\|T\left(\omega, \xi_{u}(\omega), u\right)-T\left(\omega, \xi_{v}(\omega), v\right)\right\|_{\infty} \\
\leq \max \left\{\left\|\xi_{u}(\omega)-\xi_{v}(\omega)\right\|_{E},\|u-v\|_{F}\right\} \\
=\|u-v\|_{F} .
\end{gathered}
$$

Therefore $f$ is nonexpansive on $Y$ and thus has a random fixed point $\eta: \Omega \rightarrow Y$. It follows that $T\left(\omega, \xi_{\eta(\omega)}(\omega), \eta(\omega)\right)=\left(\xi_{\eta(\omega)}(\omega), \eta(\omega)\right)$ for each $\omega \in \Omega$.

For a subset $K$ of a separable Banach space, a random operator $T: \Omega \times K \rightarrow K$ is said to be strictly contractive if

$$
\|T(\omega, x)-T(\omega, y)\|<\|x-y\|, x, y \in K, x \neq y, \omega \in \Omega \text {. }
$$

Theorem 4.2: $\quad$ Let $E$ and $F$ be two Banach spaces with $X \subset E$ and $Y \subset F$ separables. Suppose that $X$ is a closed bounded convex subset of E. Suppose also that $Y$ has the random fixed point property for strictly contractive random operators. Then for each $1 \leq P \leq \infty,(X \oplus Y)_{P}$ has the random fixed point property for strictly contractive random operators.

Proof: $\quad$ For a fixed $p, 1 \leq p \leq \infty$, suppose $T: \Omega \times(X \oplus Y)_{P} \rightarrow(X \oplus Y)_{P}$ is strictly contractive. As before, for each $y \in Y$, we define $T_{y}: \Omega \times X \rightarrow X$ by

$$
T_{y}(\omega, x)=P_{1} o T(\omega, x, y), \omega \in \Omega, x \in X .
$$

Then it is easily checked that in any case of $P, T_{y}$ is strictly contractive and hence has a random fixed point $\xi_{y}: \omega \rightarrow X[11$, Theorem 2.1]. Now define $f: \Omega \times Y \rightarrow Y$ be

$$
f(\omega, x)=P_{2} o T\left(\omega, \xi_{y}(\omega), y\right), \omega \in \Omega, y \in Y
$$

As in [25, Theorem 2.2], for any case of $p, f: \Omega \times Y \rightarrow Y$ is strictly contractive. Thus $f$ has a random fixed point $\eta: \Omega \rightarrow Y$. It follows that,

$$
T\left(\omega, \xi_{\eta(\omega)}(\omega), \eta(\omega)\right)=\left(\xi_{\eta(\omega)}(\omega), \eta(\omega)\right)
$$

for each $\omega \in \Omega$. 
Finally, we prove random fixed point theorems for nonself random operators in product spaces. Recall that for a closed subset $C$ of a separable Banach space $E$, the inward set of $C$ at a point $x$ in $C, I_{c}(x)$, is defined by

$$
I_{C}(x)=\{x+c(z-x) \mid z \in C, c>0\} .
$$

A random operator $f: \Omega \times C \rightarrow E$ is said to be weakly inward if for each $\omega \in \Omega$, $f(\omega, x) \in \operatorname{cl}\left(I_{C}(x)\right)$ for $x \in C$. We will say that $C$ has the random fixed point property for a nonexpansive (continuous) weakly inward random operator if every nonexpansive (continuous) weakly inward random operator $T: \Omega \times C \rightarrow E$ has a random fixed point.

Theorem 4.3: $\quad$ Let $E$ and $F$ be two separable Banach spaces with $X \subset E$ and $Y \subset F$. Let $E \oplus F$ be a product space with an $L^{P}$ norm, $1 \leq P<\infty$. Suppose that both $X$ and $Y$ have the random fixed point property for nonexpansive weakly inward random operators. Then $(X \oplus Y)_{P}$ also has the fixed point property for nonexpansive weakly inward random operators.

Proof: Let $T: \Omega \times(X \oplus Y)_{P} \rightarrow(E \oplus F)_{P}$ be a nonexpansive and weakly inward random operator. For a fixed $y$ in $Y$, we define $T_{y}: \Omega \times X \rightarrow E$ by

$$
T_{y}(\omega, x)=P_{1} o T(\omega, x, y), \omega \in \Omega, x \in X
$$

Then $T_{y}$ is a nonexpansive random operator. It is also weakly inward. Indeed, for each $\omega \in \Omega$. Since

$$
T(\omega, x, y) \in \operatorname{cl}\left(I_{X \oplus Y}(x, y)\right) \text { for }(x, y) \in X \times Y
$$

we have

$$
T_{y}(\omega, x)=P_{1}\left(\operatorname{cl} I_{X \oplus Y}(x, y)\right) \subseteq \operatorname{cl} I_{x}(x) .
$$

Hence there exists a measurable map $\xi_{y}: \Omega \rightarrow X$ such that for each $\omega \in \Omega$, $T_{y}\left(\omega, \xi_{y}(\omega)\right)=\xi_{y}(\omega)$. Now define $f: \Omega \times Y \rightarrow F$ by

$$
f(\omega, x)=P_{2} o T\left(\omega, \xi_{y}(\omega), y\right), \omega \in \Omega, y \in Y .
$$

Then it is easy to see that $f$ is nonexpansive. Also, for each $\omega \in \Omega, f(\omega, y) \in P_{2}(c l$ $\left.I_{X \oplus Y}\left(\xi_{y}(\omega), y\right)\right) \subseteq c l I_{y}(y)$ for $y \in Y$. Therefore, $f$ has a random fixed point $\eta: \Omega \rightarrow Y$ and hence

$$
T\left(\omega, \xi_{\eta(\omega)}(\omega), \eta(\omega)\right)=\left(\xi_{\eta(\omega)}(\omega), \eta(\omega)\right) \text { for } \omega \in \Omega
$$


In the next theorem, we assume that $X$ has the net B-G property, that is, if $T: X \rightarrow E$ is nonexpansive and if $\left\{x_{\alpha}\right\}_{\alpha \in A}$ (we also assume that $A$ is countable) is a net in $X$ for which $x_{\alpha} \rightarrow x$ weakly and $x_{\alpha}-T\left(x_{\alpha}\right) \rightarrow p$ strongly, then $x \in X$ and $x-T(x)=p$.

Theorem 4.4: $\quad$ Let $E$ and $F$ be two separable Banach spaces with $X \subset E$ and $Y \subset F$. Suppose that $X$ is weakly compact, convex, and has the net B-G property. Suppose also that $Y$ has the random fixed point property for nonexpansive weakly inward random operators. Then $(X \oplus Y)_{\infty}$ has the random fixed point property for nonexpansive weakly inward random operators.

Proof: Let $T: \Omega \times(X \oplus Y)_{\infty} \rightarrow(E \oplus F)_{\infty}$ be a nonexpansive and weakly inward random operator. then for each $y \in Y$, the operator $T_{y}: \Omega \times X \rightarrow E$ defined by $T_{y}(\omega, x)=$ $P_{1} o T(\omega, x, y),(\omega \in \Omega, x \in X)$ is nonexpansive and weakly inward. For a fixed $z \in X$ and $t \in(0,1)$, the contraction operator $(1-t) z+t T_{y}$ is weakly inward and has a unique random fixed point [Han's, 7] which we denote by $\xi_{y, t}$. Thus for $\omega \in \Omega$,

$$
\xi_{y, t}(\omega)=(1-t) z+t T_{y}\left(\omega, \xi_{y, t}(\omega)\right)
$$

Now let $\left\{t_{\alpha}: \alpha \in A\right\}$ be a universal subnet of the net $\{t: 0<t<1\}$ in $[0,1]$. It follows that $\left\{\xi_{y, t_{\alpha}}(\omega)\right\}$ is a universal net in $X\left(\xi_{y, t_{\alpha}}: \Omega \rightarrow X\right.$ is a measurable map). Also, since $t \rightarrow 1, t_{\alpha} \stackrel{\alpha}{\rightarrow} 1$ and for each $\omega \in \Omega$,

$$
\left\|\xi_{y, t_{\alpha}}(\omega)-T_{y}\left(\omega, \xi_{y, t_{\alpha}}(\omega)\right)\right\|_{E}=(1-t)\left\|z-T_{y}\left(\omega, \xi_{y, t_{\alpha}}(\omega)\right)\right\|_{E^{\stackrel{\alpha}{\rightarrow} 0} .}
$$

For each $\alpha$, define $F_{y, \alpha}: \Omega \rightarrow W K(X)$ by

$$
F_{y, \alpha}(\omega)=w^{-c l}\left\{\xi_{y, t} \alpha^{*}(\omega): \alpha^{*} \geq \alpha\right\} .
$$

Define $F_{y}: \Omega \rightarrow W K(X)$ by $F_{y}(\omega)=\bigcap_{\alpha \in A} F_{y, \alpha}(\omega)$. Then, as in the proof of Theorem 4.1, $F_{y}$ is $w$-measurable and has a measurable selector $\xi_{y}$. Furthermore, since $X$ is weakly compact, $\left\{\xi_{y, t}(\omega)\right\}$ converges weakly, say to $\xi_{y}(\omega)$. Combining this with $(*)$, the B-G property implies

$$
T_{y}\left(\omega, \xi_{y}(\omega)\right)=\xi_{y}(\omega) \text { for } \omega \in \Omega
$$

Now let $u, v \in Y, \omega \in \Omega$

$$
\begin{gathered}
\left\|\xi_{u, t}(\omega)-\xi_{v, t}(\omega)\right\|_{E}=t\left\|T_{u}\left(\omega, \xi_{u, t}(\omega)\right)-T_{v}\left(\omega, \xi_{v, t}(\omega)\right)\right\|_{E} \\
\leq t\left\|T\left(\omega, \xi_{u, t}(\omega), u\right)-T\left(\omega, \xi_{v, t}(\omega), v\right)\right\|_{\infty} \\
\leq t\left\|\left(\xi_{u, t}(\omega), u\right)-\left(\xi_{v, t}(\omega), v\right)\right\| \\
=t \max .\left\{\left\|\xi_{u, t}(\omega)-\xi_{v, t}(\omega)\right\|_{E},\|u-v\|_{F}\right\}
\end{gathered}
$$




$$
\leq t\|u-v\|_{F}
$$

Since $\left\{\xi_{u, t_{\alpha}}(\omega)-\xi_{v, t_{\alpha}}(\omega)\right\}$ converges weakly to $\xi_{u}(\omega)-\xi_{v}(\omega)$, it follows that

$$
\left\|\xi_{u}(\omega)-\xi_{v}(\omega)\right\|_{E} \leq\|u-v\|_{F} \text { for any } \omega \in \Omega .
$$

Now it is easily checked that the random operator $f: \Omega \times Y \rightarrow F$ defined by $f(\omega, y)=P_{2} o T\left(\omega, \xi_{y}(\omega), y\right), y \in Y$ and $\omega \in \Omega$ is nonexpansive and weakly inward. Thus $f$ has a random fixed point $\eta: \Omega \rightarrow Y$. It follows that $T\left(\omega, \xi_{\eta(\omega)}(\omega), \eta(\omega)\right)=\left(\xi_{\eta(\omega)}(\omega), \eta(\omega)\right)$ for each $\omega \in \Omega$.

Theorem 4.5: $\quad$ Let $E$ and $F$ be two separable Banach spaces with $X \subset E$ a closed ball with center at origin and radius $r, Y \subset F$. Let $(E \oplus F)$ be a product space with an $L^{P}$ norm $1 \leq P \leq \infty$. Suppose that $Y$ has the random fixed point property for strictly contractive weakly inward random operators. Then $(X \oplus Y)_{P}$ also has the random fixed point property for strictly contractive weakly inward random operators.

Proof: Let $T: \Omega \times(X \oplus Y)_{P} \rightarrow(E \oplus F)_{P}$ be a strictly contractive and weakly inward random operator. For a fixed $y$ in $Y$, we define the random operator $T_{y}: \Omega \times X \rightarrow E$ by $T_{y}(\omega, x)=P_{1} \circ T(\omega, x, y), \omega \in \Omega, x \in X$. Then $T_{y}$ is strictly contractive and weakly inward random operator. Hence $T_{y}$ has a random fixed point $\xi_{y}: \Omega \rightarrow X[20$, Theorem 4]. Now define $f: \Omega \times Y \rightarrow F$ by $f(\omega, y)=P_{2} o T\left(\omega, \xi_{y}(\omega), y\right)$, for $\omega \in \Omega, y \in Y$. Then it is easy to see that $f$ is strictly contractive and weakly inward random operator. Therefore, $f$ has a random fixed point $\eta: \Omega \rightarrow Y$. Hence $T\left(\omega, \xi_{\eta(\omega)}(\omega), \eta(\omega)\right)=\left(\xi_{\eta(\omega)}(\omega), \eta(\omega)\right)$ for every $\omega \in \Omega$.

Corollary 4.6: $\quad$ Let $E$ and $F$ be two Hilbert spaces with $X \subset E$ a separable closed bounded convex and $Y \subset F$ separable. Suppose $Y$ has the random fixed point property for nonexpansive and weakly inward random operators. Then $(X \oplus Y)_{2}$ also has the random fixed point property for nonself nonexpansive and weakly inward random operators.

Proof: Similar to the proof of Theorem 4.5. Only need to notice that the corresponding $T_{y}$ is nonexpansive and has the random fixed point $\xi_{y}: \Omega \rightarrow X$ from $[20$, Theorem $6]$.

\section{REFERENCES}

[1] Beg, I., Shahzad, N., Random fixed points of random multivalued operators on Polish spaces, Nonlinear Anal., to appear.

[2] Bharucha-Reid, A.T., Random Integral Equations, Mathematics in Science and Engineering Vol. 96, Academic Press, New York and London 1972. 
[3] Bharucha-Reid, A.T., Fixed point theorems in probabilistic analysis, Bull. Amer. Math. Soc. 82 (1976), 641-645.

[4] Browder, F.E., Semicontractive and semiaccretive nonlinear mappings in Banach spaces, Bull. Amer. Math. Soc. 74 (1968), 660-665.

[5] Fan, Ky, Extensions of two fixed point theorems of F.E. Browder, Math. Z. 112 (1969), 234-240.

[6] Fora, A., A fixed point theorem for product spaces, Pacific J. Math 99 (1982), 327-335.

[7] Hans, O., Reduzierende zufällige transformationen, Czechoslovak Math. J. 7 (1957), 154158.

[8] Hans, O., Random operator equations, Proc. 4th Berkeley Symposium on Math. Stat. and Prob., Vol. II, Part I, 185-202, University of California Press, Berkeley 1961.

[9] Himmelberg, C.J., Measurable relations, Fund. Math. 87 (1975), 53-72.

[10] Itoh, S., A random fixed point theorem for a multivalued contraction mapping, Pacific J. Math. 68 (1977), 85-90.

[11] Itoh, S., Random fixed point theorems with application to random differential equations in Banach spaces, J. Math. Anal. Appl. 67 (1979), 261-273.

[12] Itoh, S., Measurable or condensing multivalued mappings and random fixed point theorems, Kodai Math. J. Vol. 2, No. 3 (1979), 293-299.

[13] Khamsi, M.A., On normal structure, fixed point property and contractions of type $(\gamma)$, Proc. Amer. Math. Soc. 106 (1989), 995-1001.

[14] Kirk, W.A., Nonexpansive mappings in product spaces, set-valued mappings and $k$ uniform rotundity, Nonlinear Functional Analysis (F.E. Browder, ed.), Proc. Sympos. Pure Math. Vol. 45, Part 2, Amer. Math. Soc. (1986), 51-64.

[15] Kirk, W.A., An iteration process for nonexpansive mappings with applications to fixed point theory in product space, Proc. Amer. Math. Soc. 107 (1989), 411-415.

[16] Kirk, W.A., Yanez, C.M., Nonlinear and locally nonexpansive mappings in product spaces, Nonlinear. Anal. 12 (1988), 719-725.

[17] Kirk, W.A., Sternfeld, Y., The fixed point property for nonexpansive mappings in certain product spaces, Houston J. Math. 10 (1984), 207-214.

[18] Kuczumow, T., Fixed point theorems in product spaces, Proc. Amer. Math. Soc. 108 (1990), 727-729.

[19] Kuratowski, K., Ryll-Nardzewski, C., A general theorem on selectors, Bull. Acad. Polon. Sci. Ser. Sci. Math. Astronom. Phys. 13 (1965), 397-403.

[20] Lin, T.C., Random approximations and random fixed point theorems for nonself maps, Proc. Amer. Math. Soc. 103, (1988), 1129-1135. 
[21] Nadler, S.B., Sequences of contractions and fixed points, Pacific J. Math. 27 (1968), 579-585.

[22] Papageorgiou, N.S., Random fixed point theorems for measurable multifunctions in Banach spaces, Proc. Amer. Math. Soc. 97 (1986), 507-514.

[23] Sehgal, V.M., Singh, S.P., On random approximations and a random fixed point theorem for set-valued mappings, Proc. Amer. Math. Soc. 95 (1985), 91-94.

[24] Spacek, A., Zufällige gleichungen, Czechoslovak Math. J. 5 (1955), 462-466.

[25] Tan, K.K., Xu, H.K., On fixed point theorems of nonexpansive mappings in product spaces, Proc. Amer. Math. Soc. 113 (1991), 983-989. 


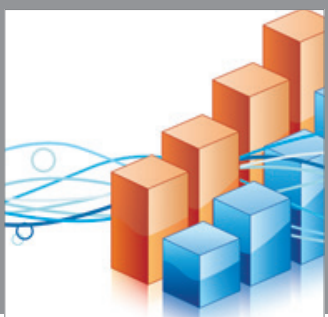

Advances in

Operations Research

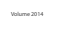

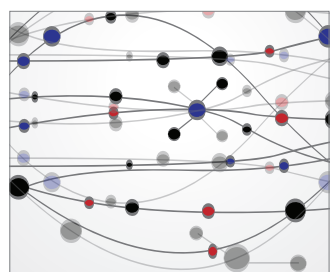

\section{The Scientific} World Journal
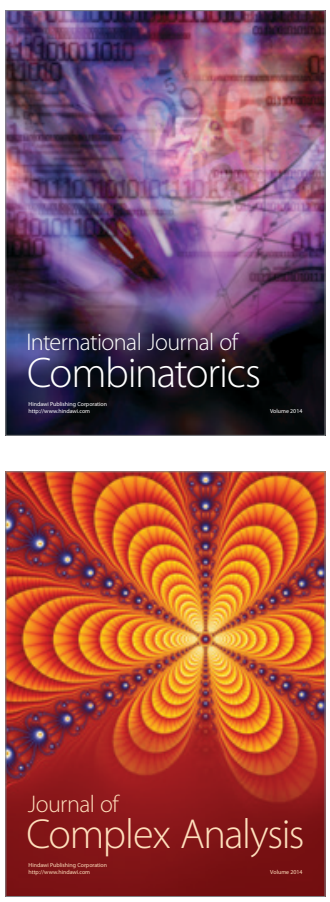

International Journal of

Mathematics and

Mathematical

Sciences
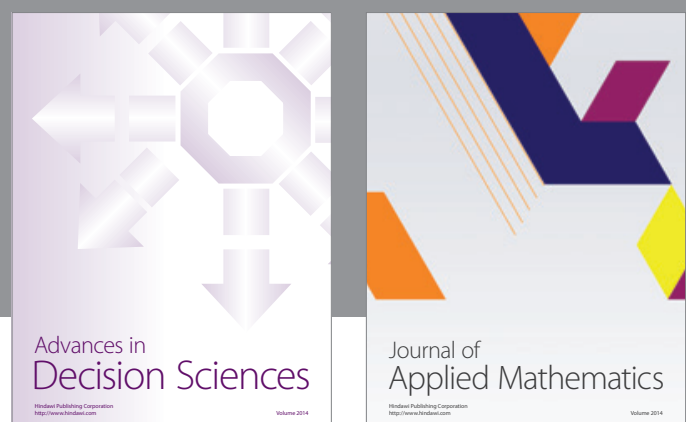

Journal of

Applied Mathematics
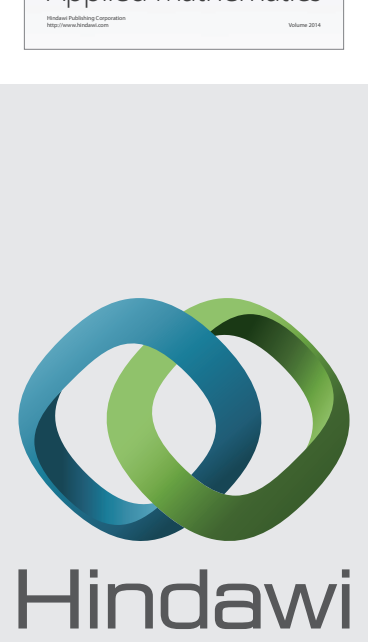

Submit your manuscripts at http://www.hindawi.com
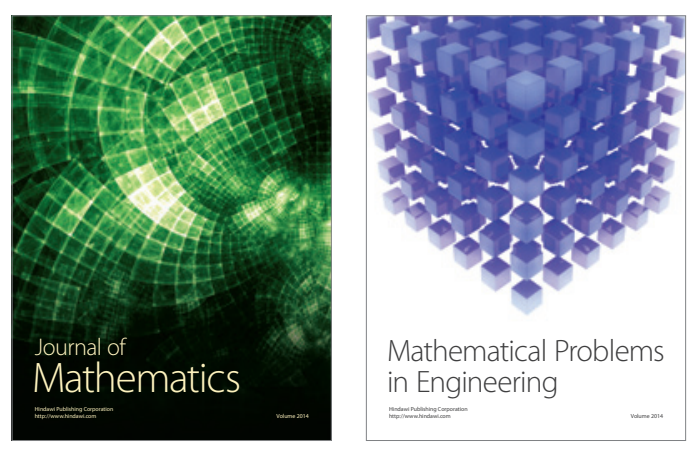

Mathematical Problems in Engineering
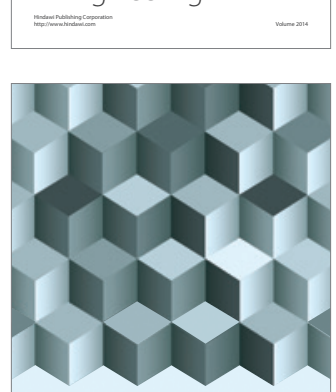

Journal of

Function Spaces
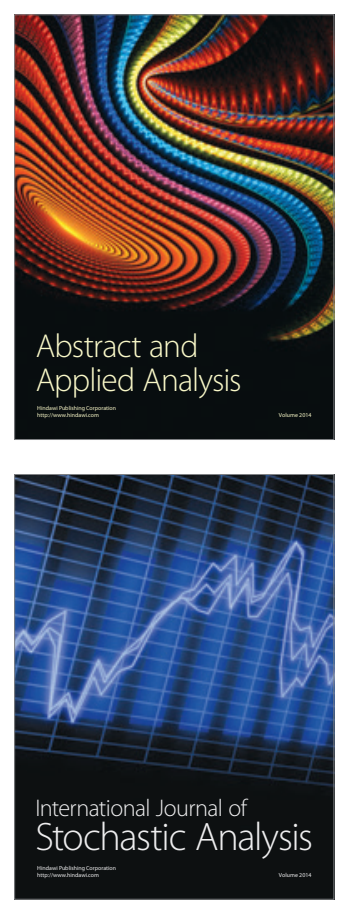

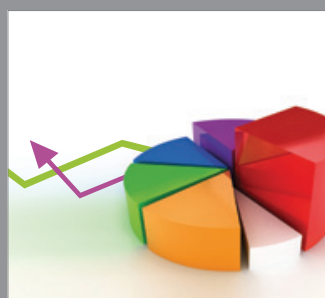

ournal of

Probability and Statistics

Promensencen
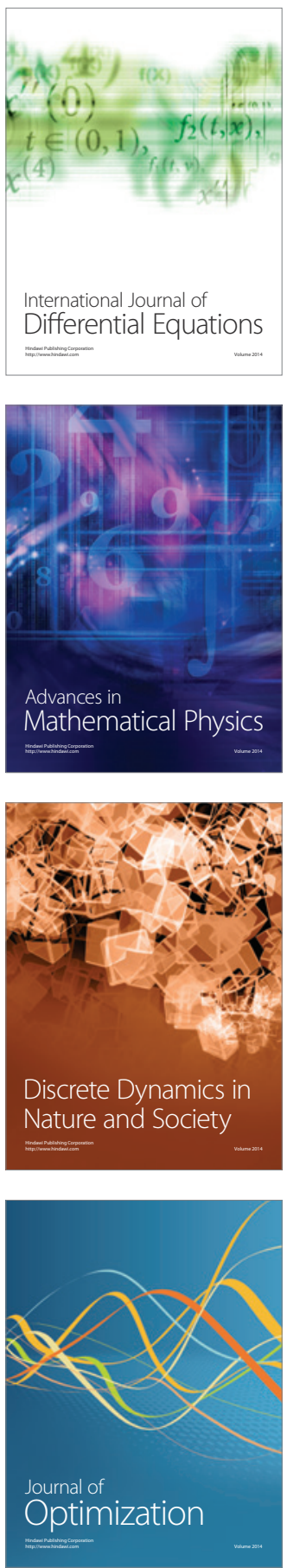